cardiovascular disease, a centralized distribution of adiposity represents a more potent risk factor than does total body obesity. To examine whether distribution of adiposity also affects the risk of dementia, Whitmer et al. conducted a longitudinal analysis of 6,583 members of the Kaiser Permanente Medical Care Program of Northern California.

Participants in the Kaiser Permanente program underwent voluntary periodic health checkups during the period 1964-1973, when they were aged $40-45$ years. At each checkup, central obesity-as measured by sagittal abdominal diameter (SAD) - was recorded. Between 1 January 1994 and 16 June 2006, some 1,049 of the original study participants were diagnosed with dementia. In multivariate models adjusted for patient demographics, diabetes, and cardiovascular comorbidities, the risk of dementia was 2.72 times greater among individuals in the highest SAD quintile than among those in the lowest quintile (95\% Cl 2.23-3.33). The addition of BMI to the model somewhat attenuated the association between central obesity and risk of dementia, but individuals in the highest SAD quintile still had a considerably greater risk for dementia than those in the lowest quintile (hazard ratio 1.98, 95\% Cl 1.33-2.32).

On the basis of these results, the authors conclude that central obesity in middle age is a risk factor for dementia even in individuals who are not overweight.

Original article Whitmer RA et al. (2008) Central obesity and increased risk of dementia more than three decades later. Neurology [doi:10.1212/01.wnl.0000306313.89165.ef]

\section{Dopamine withdrawal leads to apathy in patients with PD}

Parkinson's disease (PD) is often associated with apathy, but patients with PD who have undergone subthalamic nucleus (STN) stimulation are especially prone to this condition. It is unknown whether apathy in this population is attributable to the withdrawal of dopaminergic treatment following successful surgery or to a direct effect of STN stimulation. To investigate this issue, Czernecki et al. assessed the effect of a dopaminergic receptor agonist on apathy following STN stimulation.

The authors monitored the progress of eight patients with advanced PD who developed apathy after discontinuation of dopaminergic medication following successful surgery for STN stimulation at a hospital in Paris, France. The patients were treated for apathy with ropinirole, a dopaminergic receptor agonist with high affinity for dopamine $D_{2}$ and $D_{3}$ receptors. After 6 weeks of low-dose ropinirole treatment, the symptoms of apathy had notably diminished from baseline in seven of the eight patients. The average Starkstein Apathy scale score had decreased from $21.4 \pm 4.0$ to $9.9 \pm 6.1$, an improvement of $54 \% \pm 24 \%$. The patients' moods had also improved, as seen by a decrease in the average Montgomery-Åsberg Depression Rating Scale (MADRS) scores from $12.7 \pm 6.5$ to $4.5 \pm 8.3$, an improvement of $75 \% \pm 31 \%$; this effect was independent from the improvement in apathy.

The authors suggest that, since medical treatment successfully reversed apathy after neurosurgery despite ongoing STN stimulation, it is probable that this condition is caused by dopamine depletion or withdrawal.

Original article Czernecki V et al. (2008) Apathy following subthalamic stimulation in Parkinson disease: a dopamine responsive symptom. Mov Disord 23: 964-969

\section{Screening for early signs of autistic behaviors in ex-preterm infants}

An increased prevalence of atypical psychosocial development is seen among very-lowbirth-weight infants, but the prevalence of autism spectrum disorders in this population is unknown. Limperopoulos et al. tested 91 expreterm infants for early autistic features and identified risk factors associated with positive screening results.

Developmental outcome testing was performed in all the infants (birth weight 460$1,490 \mathrm{~g}$, gestational age at birth 23-30 weeks) at a mean age-corrected for prematurity - of $21.9 \pm 4.7$ months. In all, $23(25 \%)$ of the infants had positive results on the Modified Checklist for Autism in Toddlers (M-CHAT), and 28 (33\%) of 85 infants who underwent brain MRI (at a mean age of $39.2 \pm 3.9$ weeks) had abnormal results. Strong correlations existed between positive M-CHAT scores and the detection of behavioral problems by use of the Child Behavior Checklist, and with socialization and communication deficits as scored by the Vineland Adaptive Behavior Scales. In addition, presence of neonatal MRI abnormalities significantly predicted positive 